\section{Конструирование лесных машин на ЭВМ}

\author{
В. Н. Коршун, \\ Д. Г. Кокотов \\ Сибирский государственный технологический \\ университет
}

\begin{abstract}
АННТОЦАЦИЯ
Предложена общая методика конструирования лесных машин на ЭВМ на основе объемных моделей, метода конечных элементов, численных способов решений уравнений, получения конструкторской документации.
\end{abstract}

Ключевые слова: конструирование, объемная модель, метод конечных элементов, динамика машин.

\section{SUMMARY}

The conception method of the computer design of forest machines is presented on the basis of the Solidsimulation. The Finite-element Method and Numerical analysis are discussed as computer designing documentation.

Keywords: computer design, Solid-simulation, Finiteelement Method, machine dynamics, designing documentation

\section{ПОСТАНОВКА ЗАДАЧИ}

Целью конструирования лесных машин (ЛМ) является анализ и синтез конструктивных параметров, которые минимизируют или ограничивают последствия динамических воздействий. Конструирование при статических нагрузках позволяет создавать равнопрочные конструкции. Исследование конструкции ЛМ при динамических воздействиях позволяет решать ряд проблем: устранение пиковых напряжений от нестационарных или импульсных воздействий от взаимодействия рабочих органов с предметом труда или опорных элементов со средой движения; снижение амплитуд нагружения конструкции за счет оптимизации формы деталей; ограничение амплитуд колебаний и ускорений в заданном диапазоне частот колебаний (виброзащита); управление устойчивостью рабочих процессов и движением ЛМ. Указанные задачи решаются различными методами, в которых конструкция ЛМ представляется в виде эквивалентных расчетных схем, а элементы - в виде ограниченного числа приведенных масс, соединенных упругими связями с демпфированием [1].

\section{МЕТОДИКА КОНСТРУИРОВАНИЯ}

Развитие технических и программных средств ЭВМ позволяет конструировать ЛМ при статических и динамических нагрузках в полуавтоматизированном

\footnotetext{
${ }^{1}$ Авторы - соответственно доцент и аспирант кафедры проектирования лесного оборудования (C) В. Н. Коршун, Д. Г. Кокотов, 2003
}

режиме и получать описание технической системы в виде конструкторской документации. Задача решается следующими путями.

1. Создание объемной модели ЛМ. На основе конструкторской документации или натурных образцов компьютерными средствами создаются объемные модели деталей и сборочных единиц ЛМ, максимально соответствующие оригиналу. Опыт проектирования показывает, что наиболее удобными и наглядными средствами обладают программы SolidWorks (SolidWorks,Corp.), AutoCAD (Autodesk, Inc.) [2], Mechanical Desktop (Autodesk, Inc.) [3], ADAMS (Mechanical Dynamics, Inc.), NASTRAN (MacNealSchwendler, Corp.) [4] и др. Среди отечественных разработок, не уступающих зарубежным аналогам, отметим систему КОМПАС (АСКОН), снабженную большой библиотекой объемных моделей стандартных (ГОСТ РФ) деталей и позволяющую получать конструкторскую документацию, полностью соответствующую ЕСКД. Все названные пакеты программ обеспечивают трансляцию описания объемных моделей в международные форматы данных: ACIS (*.sat); Parasolid (текстовый или бинарный); 3D Studio и др. На объемных моделях можно определять массу, объем, моменты инерции, положения центра масс, решать несложные задачи проектирования.

2. Трансляция объемных моделей в программы конструктивных динамических расчетов. В программе COSMOS Works (Structural Research \& Analysis, Corp.), функционирующей совместно с SolidWorks; Visual Nastran Desktop (MSC.Software) [4]; ANSYS (ANSYS, Inc.); LS-DYNA (Livermore Software Tech., Corp.) и др., реализован метод конечных элементов (МКЭ) и численные алгоритмы решения уравнений статики и динамики. Указанные программные средства могут решать и задачи кинематики. Разбиение модели на конечные элементы (КЭ) и построение сетки осуществляются автоматически. Объемная конструкция ЛМ может разбиваться на линейные КЭ (стержень, труба, брус, балка, пружина), плоские КЭ (мембрана, пластина, многослойная пластина) и объемные КЭ (тела), с плоскими или криволинейными поверхностями. Для КЭ задается материал (из библиотеки) с изотропными или анизотропными свойствами. Все свойства КЭ можно задавать в виде зависимостей или из файла данных.

Уравнения динамики ЛМ в общем виде можно представить формулой

$$
M \cdot[\cdot \ddot{x}]+K \cdot[\dot{x}]+\mathbf{F} \cdot \mathbf{F}=\mathbf{F}(t)_{-}^{-}
$$

где $[M]$ - матрица инерционности конструкции (масс и моментов инерции); $[K]-$ матрица коэффициентов сил вязкого демпфирования, пропорциональных скоростям; $[C]$ - матрица жесткости; $[F(t)]$ - вектор нагрузок как функция времени, $[x]$ - вектор переме-

щений (деформаций); $x, x$ - первая и вторая производные от перемещений соответственно. 
Матрица масс включает в себя: массы КЭ, обусловленные плотностью задаваемого материала; дополнительные (неконструктивные) массы КЭ; сосредоточенные (точечные) массы. Матрица $[K]$ учитывает диссипацию энергии в динамической системе посредством задания: демпфирующих свойств материалов коэффициентом $\mathrm{K}_{д}=2 \cdot \mathrm{K} / \mathrm{K}_{0}(\mathrm{~K}-$ коэффициент пропорциональности для силы вязкого демпфирования как функции скорости, $\mathrm{K}_{0}$ - коэффициент критического демпфирования, при котором колебательная форма движения сменяется монотонно затухающей (по экспоненте)); коэффициентом демпфирования для элементов типа многослойных пластин или комбинаций пружин (жесткой) и демпферов; общего коэффициента (конструктивного) демпфирования.

Уравнения (1) дополняются начальными условиями

$[x(0)]=x 0, \quad[\dot{x}(0)]=x 0$,

где $x 0, x 0-$ векторы перемещений и скоростей в начальный момент времени $(t=0)$.

При задании нагрузок при конструировании на ЭВМ учитывают специфику работы ЛМ. Большинство программных систем позволяют задавать следующие виды нагрузок: силы и моменты (Force/Moments), сосредоточенные и распределенные по длине и поверхности; кинематические (несиловые) воздействия (ускорения, скорости и перемещения); тепловые воздействия (температура, тепловыделение, тепловой, конвективный и радиационный потоки). К самим моделям нагрузки могут прикладываться по объему (гравитация, центробежные и силы инерции), к узлам (силы), к КЭ (в точке, на линии и на поверхности). Нагрузки могут задаваться в виде выражений, зависящих от параметров динамической системы, или вводиться из файла данных. Нелинейные нагрузки, зависящие от перемещения или скоростей КЭ, используются для моделирования нестационарных процессов. Нагрузки от рабочих органов и опорных элементов ЛМ моделируются как стационарные с единичными импульсными воздействиями от единичных включений в предмете труда и препятствий в среде движения. На моделях также указываются ограничения (связи).

\section{МЕТОДЫ РЕШЕНИЯ}

Для решение уравнений (1) и (2) в компьютерных программных системах используются различные методы: непосредственное численное интегрирование по времени уравнений (1) при начальных условиях (2) (Direct Transient); разложение вектора перемещений $[x]$ в ряд по формам собственных колебаний (без демпфирования) (Modal Transient) для переходных процессов; частотный анализ (Frequency) при гармоничном законе движения; быстрое преобразование Фурье (FFT); быстрый нелинейный анализ (FNA) (метод Вильсона) для динамических систем с предопределенными физическими или геометрическими ограниченными нелинейностями (шарниры с зазорами, подвеска с нелинейными показателями, сухое трение) (SAP2000). Программные средства могут передавать информацию для расчетов в другие системы, в том числе и математические MathCAD и MATLAB.

\section{ВЫВОД РЕЗУЛЬТАТОВ}

Все указанные программные средства позволяют выводить для конструктивного анализа перемещения, напряжения, деформации геометрии структур, нагрузки и внутренние силовые факторы. Для первоначального анализа данные выводятся в виде трехмерных или плоских изображений с цветной заливкой, соответствующей величине результата, или в виде анимации деформированных форм и очертаний формы колебаний модели. Кроме расчетов системы NASTRAN, ANSYS позволяют проводить оптимизацию для задач статики, установившихся и переходных динамических процессов, расчеты долговечности, собственных частот и форм колебаний, тепловых процессов. В результате динамического анализа получается оптимальная объемная модель ЛМ. На основе объемной модели ЛМ формируется конструкторская документация в виде чертежей.

\section{ЗАКЛЮЧЕНИЕ}

Компьютерное конструирование на начальных стадиях создания ЛМ без значительных материальных затрат в многовариантном режиме позволяет проводить анализ и синтез кинематических и динамических параметров, выполнять прочностные расчеты, оптимизировать марки материалов, форму, структуру и геометрию конструкции, оценивать поведение системы при различных внешних воздействиях, определять безрезонасные режимы работы и параметры виброзащиты, выводить результаты расчетов и конструктивных решений в виде конструкторской документации.

\section{СПИСОК ЛИТЕРАТУРЫ}

1. Александров В. А. Моделирование технологических процессов лесных машин. М.: Экология, 1995. $257 \mathrm{c}$.

2. Коршун В. Н. Основы автоматизированного проектирования: Объемное конструирование деталей на основе редактора AutoCAD: Учебное пособие. Красноярск, 2002. 128 с.

3. Дэниел Т. Банах. Mechanical Desktop 4. М: Лори, $2001.526 \mathrm{c}$.

4. Шимкович Д. Г. Расчет конструкций в MS/NASTRAN for Windows. М.: ДМК Пресc, $2001.448 \mathrm{c}$. 